\title{
Hybridization of Evolutionary Computation Techniques for Job Scheduling Problem
}

\author{
V.Selvi \\ Associate Professor, \\ Department of M.C.A, \\ M.A.M.College of Engineering, \\ Siruganur,Trichy. Tamilnadu. India.
}

\author{
R.Umarani \\ Associate Professor, \\ Department of Computer Science \\ Sri Saradha College for Women \\ Salem. Tamilnadu, India.
}

\begin{abstract}
In the field of computer science and operation's research, PSO is an optimization algorithm which is inspired by social behaviour of bird flocking and fish schooling. The original PSO was used to solve continuous optimization problems. Crossover and mutation of the particle are modified due to the discrete solution's spaces of scheduling optimization problems. Artificial Bee Colony $(\mathrm{ABC})$ is an optimization algorithm relatively new swarm intelligence technique based on behaviour of honey bee swarm and Meta heuristic. It is successfully applied to various paths mostly continuous optimization problems. Swarm intelligence systems are typically made up of a population of simple agents or boids interacting locally with one another and with their environment. The job scheduling problem is the problem of assigning the jobs in the system in a manner that will optimize the overall performance of the application, while assuring the correctness of the result. PSO and ABC algorithm is proposed in this paper, for solving the job scheduling problem with the criterion to decrease the maximum completion time. In this paper, modifications to the PSO and ABC algorithm is based on Genetic Algorithm (GA) of crossover and mutation operators. Such modifications applied to the creation of new candidate solutions improved performance of the algorithm.
\end{abstract}

Keywords: Particle Swarm Optimization, Artificial Bee Colony, Genetic algorithm, Job scheduling

\section{INTRODUCTION}

Scheduling jobs has been a popular research topic for many years. There are many different ways to schedule jobs and the threads which make them up. However, only a few mechanisms are used in practice and studied in detail[1]. Users submit jobs in batch to a resource management system queue and a centralized scheduler decides how to prioritize and allocate resources for job execution[10]. To minimize the response time, the scheduling system strategy needs to prioritize competing user jobs with varying levels of priorities and importance and allocate resources accordingly[2]. Several inhabitants based on an algorithm have been proposed to find near-optimal solutions to the difficult optimization problems like scheduling and routing problems. An inhabitant based algorithm refers the inhabitants consisting of possible solutions to the problem are modified by applying some operators on the solutions depending on the information of their fitness values. Several heuristic traditional algorithms were used for solving the job scheduling problem based on an algorithm are classified into Genetic algorithm (GA), Particle Swarm Optimization (PSO) algorithm and Artificial Bee Colony Algorithm.

The optimization methods based on the concept of swarm intelligence have been highlighted in the literature. These methods are based on the behaviour of social intelligence of groups of insects or animals that have characteristics of selforganization and decentralized control, with multiple agents. PSO shares many similarities with evolutionary computation techniques such as Genetic Algorithms (GA). The system is initialized with a population of random solutions and searches for optima by updating generations. However, unlike GA, PSO has no evolution operators such as crossover and mutation. In PSO, the potential solutions, called particles, fly through the problem space by following the current optimum particles. Another reason that PSO is attractive is that there are few parameters to adjust. One version, with slight variations, works well in a wide variety of applications. Particle swarm optimization has been used for approaches that can be used across a wide range of applications, as well as for specific applications focused on a specific requirement. In past several years, PSO has been successfully applied in much research and application areas. It is demonstrated that PSO gets better results in a faster, cheaper way compared with other methods. Artificial Bee Colony Algorithm belongs to this class. It should be emphasized that this method belongs to the metaheuristic class, which are an alternative option when the deterministic or more traditional methods experience difficulties. As a relatively new member of swarm intelligence, an artificial bee colony (ABC) algorithm is based on collective behaviour of self-organized systems. So far, $\mathrm{ABC}$ algorithm has received interest from researchers in a variety of fields. The $\mathrm{ABC}$ algorithm was first proposed to optimize multi-variable and multi-modal continuous functions. Many comparative studies showed that the performance of the $\mathrm{ABC}$ algorithm was competitive when compared to other population-based algorithms with the advantage of employing fewer control parameters in the continuous

space.

This paper performs an experiment on proposed Adaptive $\mathrm{ABC}$ with crossover and mutation operations to find best food source positions. After the crossover and mutation operation, the fitness of the individual food source's best position is compared with that of the two off-springs, and the best one is taken as the new individual best food source position. Two food sources are selected as parents through selection process and calculate their fitness values. After 
selecting crossover and mutation points randomly, new fitness values are generated using crossover and mutation's probabilities. Both the above techniques perform a crossover and mutation by swapping the food source around the crossover and mutation points.

The remainder of this paper is organized as follows: Section 2 briefly reviews the Genetic algorithm. Section 3 presents the basic PSO algorithm and discusses the modified PSO technique. Section 4 presents the basic $\mathrm{ABC}$ algorithm and discusses the proposed adaptive $\mathrm{ABC}$ technique, which is detailed in its subsections. Section 5 discusses about the Experimental Results and Discussion. Section 6 concludes the paper.

\section{GENETIC ALGORITHM}

In the computer science field of artificial intelligence, Genetic Algorithm (GA) is a search heuristic that mimics the process of natural evolution. This heuristic is routinely used to generate useful solutions to optimization and search problems. Basically, it consists of five components: a random number of generator, a fitness evaluation unit and genetic operators for reproduction; crossover and mutation operations.[13]

\section{Simple generational genetic algorithm procedure}

\section{Choose the initial population of individuals \\ 2. Evaluate the fitness of everyone in that population. \\ 3. Repeat on this generation until termination (time limit, sufficient fitness achieved, etc.): \\ $i$. Select the best-fit individuals for reproduction ii. Breed new individuals through crossover and mutation operations to give birth to offspring iii. Evaluate the individual fitness of new individuals \\ 4. Replace least-fit population with new individuals}

Figure 1: The procedure of Genetic algorithm

The initial population required at the start of the algorithm, is a set of food source generated by the random generator. Each position of a food source represents a possible solution of the optimization problem, and the nectar amount of a food source corresponds to the quality (fitness) of the associated solution. A fitness value is a measure of the goodness of the solution that it represents. Essentially the aim of the genetic operators is to transform this set of food source into sets with superior fitness values. The reproduction operator performs a natural selection function known as seeded selection. The crossover and mutation operator chooses pairs of food sources at random and produces new pairs. The simplest crossover and mutation operation is to cut the original food sources nectar amount at a randomly selected point and to exchange. The number of crossover and mutation operations is governed by a crossover and mutation rate. The mutation operator randomly mutates or reverses the values of food source. The number of mutation operations is determined by a mutation rate[14][16]. A phase of the algorithm consists of applying the evaluation, reproduction, crossover and mutation operations.

\subsection{Genetic algorithm for Job scheduling}

Step 1. Initialize the population as input number of processors, number of jobs.

Step2. Process started

Step2.1 Evaluate the fitness function (makes pan).

Step2. 2. Perform selection to select best individuals from the current population.

Step2. 3. Perform two-point crossover. Choose pairs of chromosomes (task). Choose a random point exchange machine assignments from that point until the end of the chromosome.

Step 2.4.Mutation: Randomly select a task.

Randomly, reassign it to the new machine.

Step 3. The process is repeated until the stopping criterion is met. (Best fitness, minimum completion time)

Step 4. Stop

Figure 2: The procedure of genetic algorithm for job scheduling

\section{PARTICLE SWARM OPTIMIZATION}

Particle swarm optimization (PSO) is a population based stochastic optimization technique, inspired by social behaviour of bird flocking or fish schooling. Each particle keeps track of its coordinates in the problem spaces which are associated with the best solution (fitness) it has achieved so far. (The fitness value is also stored.) This value is called pbest. Another "best" value that is tracked by the particle swarm optimizer is the best value, obtained so far by any particle in the neighbours of the particle. This location is called lbest. When a particle takes all the population as its topological neighbours, the best value is a global best and is called gbest.

After finding the two best values, the particle updates its velocity and positions with following equation (a) and (b).

v[ $=v \square+c 1 *$ rand $0 *$ (pbest $\square-$ pressent [)$+c 2 *$ rand $0 *$ (gbest $\square-$ present $\square--(a)$ present []$=$ present $[\square+v[]-\ldots-(b)$

$v[]$ is the particle velocity, present[] is the current particle (solution). pbest[] and gbest[] are defined as stated before. rand () is a random number between $(0,1), \mathrm{c1}, \mathrm{c2}$ are learning factors. Usually $\mathrm{c1}=\mathrm{c} 2=2$.

The pseudo code of the procedure is as follows.

For each particle

Initialize particle

END

Do

For each particle

Calculate fitness value

If the fitness value is better than the best fitness value (pBest) in history End set current value as the new pBest

Choose the particle with the best fitness value of all the particles as the gBest

For each particle 
Calculate particle velocity according to equation--- (a)

Update particle position according to equation ----(b) End

While maximum iterations or minimum error criteria is not attained

Particles' velocities on each dimension are clamped to a maximum velocity Vmax. If the sum of accelerations would cause the velocity on that dimension to exceed Vmax, which is specified by the user. Then the velocity on that dimension is limited to Vmax.

\subsection{Modified Particle Swarm Optimization for Scheduling}

Step1: Initialize swarm size and particle values for each particle ( Initialize the number of resources and number of jobs)

Step2: Choose the particle with the best fitness value of all the particles as the gbest

Do

Step 3: For each particle: (generate a new fitness value by using the crossover and mutation operations.)

Step3.1 Perform two-point crossover for a Job. Choose a random point exchange machine assignments. Step 3.2 Mutation: Randomly select a job. Randomly, reassign it to the new machine.

Step 4: Update particle velocity:

Step 5: Calculate particle velocity

Step 6: Update particle position End

while maximum iterations or minimum error criteria are not attained (or the process is repeated until the stopping criterion is met. (Best fitness, minimum completion time)

Figure 3: The procedure of Modified Particle Swarm Optimization algorithm

\section{THE ARTIFICIAL BEE COLONY ALGORITHM}

Inspired by the intelligent foraging behaviours of Honeybee Swarm, an Artificial Bee Colony algorithm is developed, which is a new population-based meta-heuristic approach. In $\mathrm{ABC}$ algorithm, three different kinds of foraging bees are involved: employed bees, onlooker bees, and scout bees. The procedure that followed in the $\mathrm{ABC}$ algorithm is given in Fig. 3.

Initialization phase

Step 1: Initialize parameters, including the number of food sources or the number of employed bees, number of onlooker bees and number of scout bees.

Step 2: Initialize population of food sources with random solutions.
Step 3: Calculate the objective value of each food source and then determine the best food resource.

Employed bee phase

Step 4: For every employed bee, generate a new food source.

Step 5: Calculate the objective value for every new food sources and compute the best food source.

Onlooker bee phase

Step 6: Calculate the probability of selecting food source using Equ. (1).

Step 7: Calculate the number of onlooker bees to be sent to the food source.

Step 8: Every onlooker bee, generate the new food sources.

Scout bee phase

Step 9: Initialize scout bees with random solutions and update the best food sources.

Step 10: Determine the worst employed bees and replace them with the scout bees if the scout bees are better.

Step 11: If a stopping criterion is met, then the best food source is obtained with its objective value.

Figure 4: The basic procedure of $\mathrm{ABC}$ algorithm

Employed bees are those bees that are exploiting a food source currently. The working process of the bees is described as follows:

The employed bees bring loads of nectar from the food sources to the hive and then share the food source information with onlooker bees by dancing in a common area in the hive called dance area. The duration of a dance is proportional to the nectar content of the food source currently being exploited by the dancing bee. Onlooker bees need to watch numerous dances before choosing a food source, which tends to choose a food source according to the probability proportional to the quality of that food source. Therefore, the good food sources tend to attract more bees than the bad ones. A scout or onlooker bee may change into an employed bee when it finds a better source. An employed bee associated with a food source may become a scout or onlooker bee when the food source is exploited fully.

In the $\mathrm{ABC}$ algorithm, each food source represents a possible solution to the problem under consideration, and the nectar amount of a food source represents the quality of the solution. The ABC algorithm assumes that there is only one employed bee for every food source. i.e. The number of food sources is same as the number of employed bees. The employed bee of an abandoned food source becomes a scout bee and as soon as it finds a new food source, it becomes an employed bee again. The $\mathrm{ABC}$ algorithm is an iterative algorithm. It starts by associating all employed bees with randomly generated food 
sources (solution). Then, every employed bee moves to a new food source in the neighbourhood of its currently associated food source and evaluates its nectar amount (objective value) during iterations. When the employed bees complete the process, they share the nectar information of the food sources with the onlooker bees and the number of onlooker bees to be sent to the food source found by the employed bee is proportional to the nectar amount of that food source.

The probability of selecting food sources $i$ is determined by the following expression:

$$
p_{i}=\frac{f_{i t}{ }_{i}}{\sum_{i=1}^{l} f i t_{i}}
$$

Here, $f i t_{i}$ is the objective value of the solution represented by the food sources, $i$ and $l$ is the total number of food sources. Clearly, good food sources will attract more onlookers than the bad ones.

\subsection{Adaptive ABC Algorithm}

In adaptive $\mathrm{ABC}$ algorithm, new food sources are generated by accomplishing the crossover and mutation operations. The crossover and mutation are the genetic algorithm operations. In this proposed method, crossover and mutation operator is added after the employed bee phase of Artificial Bee Colony algorithm. ABC algorithm has four phases namely initialization phase, employed bees phase, onlooker bee's phase and scout bees phase, adding mutation phase after the employed bee phase. Employed bee phase do the local search and mutation after the employed bee phase explore the search space and search for new area of solution space. Through mutation, on the one side, there is a chance of changing the local best position and the algorithm may not be trapped into local optima. On the other side, individual can make use of the others advantage by sharing information mechanism. In this method, the mutation step is carried out on the probabilistic way in each food searching operation for each iteration during the life cycle of $\mathrm{ABC}$ optimization technique. Food Source is selected arbitrarily from the food size and mutation is performed. In mutation, generated offspring's replaces the older offspring's. The mutation operator used in this paper is uniform mutation. When performing mutation, food source $\mathrm{x}_{\mathrm{ij}}$ is randomly selected and replaces it's one of the dimension values by random number generated in between the lower and upper bound value of the food source.

The procedure that followed in the adaptive $\mathrm{ABC}$ algorithm is demonstrated in Fig. 4.

\section{Initialization phase}

Step 1: Initialize input parameters, including the number of food sources or the number of employed bees $e_{b}$, number of onlooker bees $O_{b}$ and number of scout bees $S_{b}$.

Step 2: Initialize populations by generating random food sources $f_{S}$.

Step 3: Calculate the fitness value $F\left(f_{S}\right)$ of each food source $f_{s}$ and then determine the best food resource $b f_{s}$. Employed bee phase

Step 4: For every employed bee, generate a new food source $N f_{s}\left(e_{b}\right)$ by using the crossover and mutation operations.

Step 5: Calculate fitness value $F\left(f_{s}\left(e_{b}\right)\right)$ for every newly generated food sources and compute the best food source.

Onlooker bee phase

Step 6: For every onlooker bee, generate a new food source $N f_{s}\left(o_{b}\right)$ by using the crossover and mutation operations.

Step 7: Calculate fitness value $F\left(f_{s}\left(o_{b}\right)\right)$ for every newly generated food sources and compute the best food source.

Scout bee phase

Step 9: Initialize scout bees with random solutions and compute fitness value $F\left(f_{s}\left(s_{b}\right)\right)$ for these random solutions.

Step 10: Find the best scout bee among the randomly generated food sources using the fitness value $F\left(f_{s}\left(s_{b}\right)\right)$.

Step 11: The scout bee's best food source $B\left(f_{s}\left(s_{b}\right)\right)$, the employed bee's best food source $B\left(f_{s}\left(s_{b}\right)\right)$ and the onlooker bee's best food source $B\left(f_{s}\left(s_{b}\right)\right)$ are compared based on their fitness values.

Step 12: Among these food sources, the best food source is stored in the scout bee's phase and remaining food sources are given to the next iteration.

Step 13: The process is repeated until the stopping criterion is met. Then, the best food source is obtained with its objective value from the scout bee's phase.

Figure 5: The procedure of Adaptive ABC algorithm

\subsection{Proposed Adaptive ABC technique for Job Scheduling}

The proposed technique finds out the way for the assignment of jobs to the resources by achieving minimum completion time. The adaptive $\mathrm{ABC}$ algorithm is initiated by generating food sources $f_{S}$. The food sources are represented as $f_{s i}=\left\{R_{1}, R_{2}, \cdots R_{k}\right\} ; k \in M$, where $i$ represents the number of generated food sources. In this food source, each resources have the allocated jobs based on their execution and processing times $e_{n}$ and $p_{n, m}$.

\subsubsection{Crossover and mutation in the employed bee phase}

In our $\mathrm{ABC}$ algorithm, employed bees perform global exploration with multiple different neighbourhoods for promising food sources over the entire region. Since the JSP consists of machine assignment and operation sequence, we will design the crossover and mutation operators to evolve the machine assignment and operation sequence. 
4.2.2 Crossover and mutation for machine assignment

To evolve the machine assignment, two crossover and mutation operators are applied with equal probability, i.e., the two-point crossover and mutation and uniform crossover and mutation. These crossover and mutation operators only change the machine assignment but not change the operation sequence. To the two feasible parents, the offspring generated by these crossover and mutation operators is still feasible

\subsubsection{Mutation for machine assignment}

To enhance the exploration capability in the employed bee search phase, a mutation operator for machine assignment is proposed and embedded in the $\mathrm{ABC}$ algorithm. To reduce the computation load, the following mutation procedure is used in the $\mathrm{ABC}$ algorithm with a probability $50 \%$ :

Step 1. Randomly generate an integer I from 1 to $n$, where $n$ is the total number of operations

Step 2. Randomly select I positions from the machine assignment vector

Step 3. For each selected position, replace the machine with a different machine randomly chosen from the candidate machine set (no change happens if the set only includes one machine)

\section{EXPERIMENTAL RESULTS AND DISCUSSION}

The performance of the proposed algorithm is evaluated under five different job datasets and the results are compared against the conventional algorithms such as PSO, ABC, GA, PSO_GA and ABC_GA. The results that are obtained in different experiments are given in following Table I.

Table 1: Job Completion Time for Job Dataset I by the proposed with (i) 50 iterations, (ii) 100 iterations and (iii) 200 iterations PSO, ABC, GA, PSO_GA and ABC_GA algorithm with the number of 10 jobs and resources are 50,100,150,200.

\begin{tabular}{|c|c|c|c|c|c|c|c|}
\hline $\begin{array}{l}\text { No } \\
\text { of } \\
\text { jobs }\end{array}$ & $\begin{array}{l}\text { No of } \\
\text { Resour } \\
\text { ce }\end{array}$ & $\begin{array}{l}\text { Test } \\
\text { Run } \\
\mathrm{s}\end{array}$ & PSO & ABC & GA & $\begin{array}{l}\mathrm{PSO}_{-} \\
\mathrm{GA}\end{array}$ & $\begin{array}{l}\mathrm{ABC}- \\
\mathrm{GA}\end{array}$ \\
\hline 10 & 50 & 50 & 220 & 180 & 190 & 162 & 160 \\
\cline { 3 - 8 } & & 100 & 230 & 210 & 185 & 265 & 161 \\
\cline { 3 - 8 } & 200 & 215 & 225 & 160 & 224 & 127 \\
\hline 10 & 100 & 50 & 220 & 240 & 210 & 237 & 224 \\
\cline { 3 - 8 } & & 100 & 217 & 320 & 222 & 237 & 227 \\
\cline { 3 - 8 } & 200 & 192 & 315 & 218 & 185 & 183 \\
\hline 10 & 150 & 50 & 120 & 190 & 150 & 110 & 96 \\
\cline { 3 - 8 } & & 100 & 105 & 180 & 112 & 100 & 98 \\
\cline { 3 - 8 } & & 200 & 90 & 160 & 98 & 78 & 74 \\
\hline 10 & 200 & 50 & 117 & 220 & 120 & 117 & 110 \\
\cline { 3 - 8 } & & 100 & 120 & 210 & 110 & 130 & 99 \\
\cline { 3 - 8 } & & 200 & 115 & 180 & 110 & 104 & 91 \\
\hline
\end{tabular}

The results that are obtained in different experiments are given in following Figures 6 to 9 .

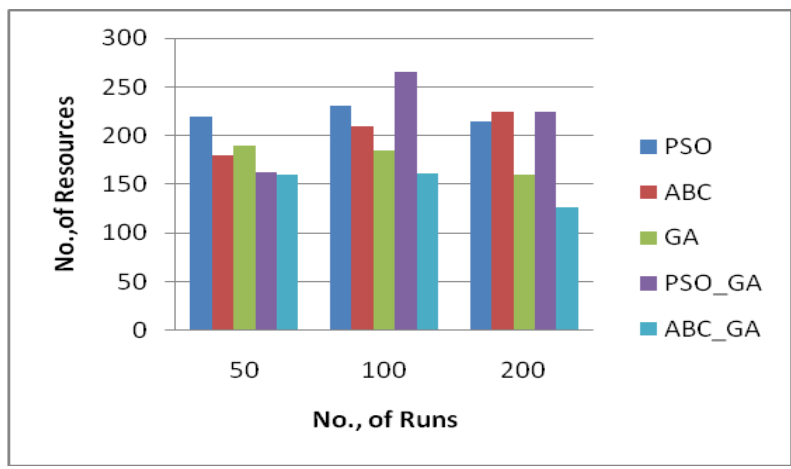

Figure 6: Mean Job Completion Time for individual Job Datasets proposed with 50 iterations, PSO, ABC, GA, PSO_GA and ABC_GA

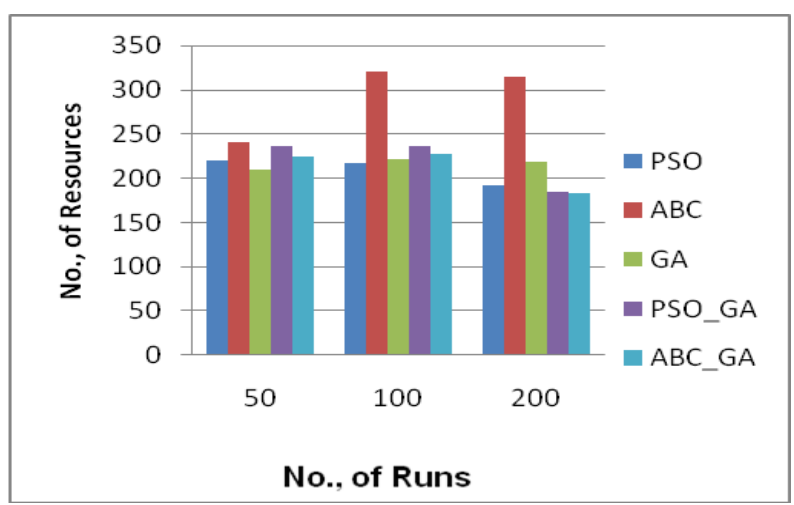

Figure 7: Mean Job Completion Time for individual Job Datasets proposed with 50 iterations PSO, ABC, GA, PSO_GA and ABC_GA.

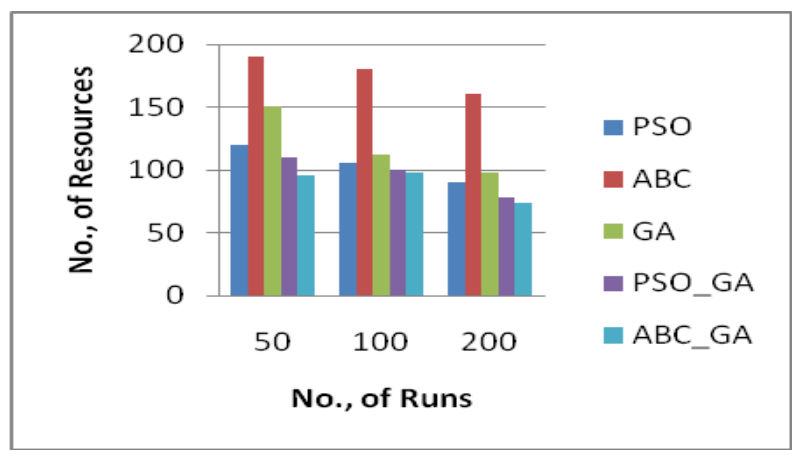

Figure 8: Mean Job Completion Time for individual Job Datasets proposed with 50 iterations PSO, ABC, GA, PSO_GA and ABC_GA. 


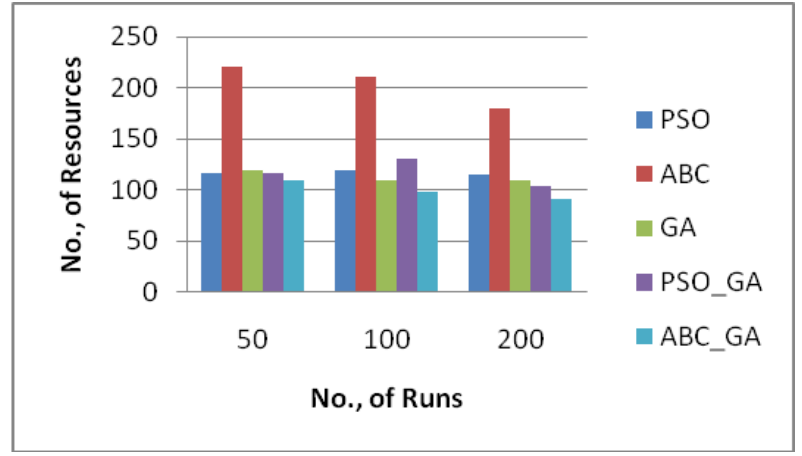

Figure 9: Mean Job Completion Time for individual Job Datasets proposed with 50 iterations PSO, ABC, GA, PSO_GA and ABC_GA.

\section{CONCLUSION}

In this paper, the proposed method has achieved the minimum completion and makes span time. The drawbacks of existing techniques were solved by considering some efficient factors in the job scheduling process. Thus, the proposed technique has achieved high performance in allocating the available jobs to the precise resources and also attained a high efficiency. The performance of the proposed job scheduling technique was analyzed with three hybrid techniques namely PSO, ABC and GA with experimental results that prove that the proposed job scheduling technique has attained high accuracy and efficiency than the three hybrid techniques. Hence, the proposed Adaptive $\mathrm{ABC}$ job scheduling technique is capable of finding the optimal jobs to the resources and also achieving the minimum completion time.

\section{REFERENCES:}

[1] Dror G. Feitelson, Larry Rudolph and Uwe Schwiegelshohn, "Parallel Job Scheduling -A Status Report", In Proeedings of the Conference on JSSPP, pp.1-16, 2004.

[2] Ivan Rodero, Francesc Guim and Julita Corbalan, "Evaluation of Coordinated Grid Scheduling Strategies", In Proceedings of 11th IEEE International Conference on High Performance Computing and Communications, Seoul, pp. 1-10, 2009.

[3] Oliner, Sahoo, Moreira, Gupta and Sivasubramaniam, "Fault-aware Job Scheduling for BlueGene/L Systems", In Proceedings of 18th International Parallel and Distributed Processing Symposium, 2004.

[4] Grudenic and Bogunovi, "Computer Cluster Scheduling Algorithm Based on Time Bounded Dynamic Programming", In Proceedings of the 34th International Convention on MIPRO, 2011, Opatija, pp. 722-726, 2011

[5] Abdelrahman Elleithy, Syed S. Rizvi and Khaled M. Elleithy, "Optimization and Job Scheduling in Heterogeneous Networks ", International Joint Conferences on Computer, Information, and Systems Sciences, and Engineering, 2008
[6] Zhang, Franke, Moreira and Sivasubramaniam, "A Comparative Analysis of Space- and Time-Sharing Techniques for Parallel Job Scheduling in Large Scale Parallel Systems", pp. 1-33, 2008.

[7] Surekha and Sumathi, "Solution to the Job Shop Scheduling Problem using Hybrid Genetic Swarm Optimization Based on $(\lambda, 1)$-Interval Fuzzy Processing Time", European Journal of Scientific Research, Vol. 64, No. 2, pp. 168-188, 2011.

[8] [8]. Bin Cai, Shilong Wang and Haibo Hu, "Hybrid Artificial Immune System for Job Shop Scheduling Problem", World Academy of Science, Engineering and Technology, Vol. 59, No. 18, pp. 81-86, 2011.

[9] Mohammad Akhshabi, Mostafa Akhshabi and Javad Khalatbari, "Parallel Genetic Algorithm to Solving Job Shop Scheduling Problem", Journal of Applied Sciences Research, Vol. 1, No. 10, pp. 1484-1489, 2011.

[10] Manish Gupta, Govind sharma, "An Efficient Modified Artificial Bee Colony Algorithm for Job Scheduling Problem", International Journal of Soft Computing and Engineering (IJSCE), Vol. 1, No. 6, pp. 291-296, January 2012.

[11] Hadi Mokhtari, "Adapting a Heuristic Oriented Methodology for Achieving Minimum Number of Late Jobs with Identical Processing Machines", Research Journal of Applied Sciences, Engineering and Technology, Vol. 4, No. 3, pp. 245-248, 2012.

[12] Elnaz ZM, Amir MR, Mohammad R, Feizi D (2008). Job Scheduling in Multiprocessor Architecture Using Genetic Algorithm. Proc. IEEE, pp. 248-250.

[13] Thanushkodi K, Deeba K (2009). An Evolutionary Approach for Job Scheduling in a Multiprocessor Architecture. CiiT Int. J. Artif. Intell. Syst. Mach. Learn., 1(4).

[14] Tung-Kuan L, Jinn- Tsong T, Jyh-Hong C (2005). Improved genetic algorithm for the job-shop scheduling problem. International Journal Advanced Manufacture Technology (Spiringer), pp. 1021-1029.

[15] D. Y. Sha and Hsing-Hung Lin A multi-objective PSO for job-shop scheduling problems Expert Systems with Applications, Volume 37, Issue 2, March 2010, Pages 1065-1070.

[16] K. Thanushkodi, K. Deeba, On Performance Analysis of Hybrid Algorithm (Improved PSO with Simulated Annealing) with GA, PSO for Multiprocessor Job Scheduling, ISSN: 1109-2750 287 Issue 9, Volume 10, September 2011.

[17] Kao, Ming-Hsien Chen, and Yi-Ting Huang, Research Article A Hybrid Algorithm Based on ACO and PSO for Capacitated Vehicle Routing Problems, Mathematical Problems in Engineering, Volume 2012.

[18] Deepak Singh, Ankit Sirmorya, Solving Real Optimization Problem using Genetic Algorithm with Employed Bee (GAEB),International Journal of Computer Applications (0975 - 8887) Volume 42No.11, March 2012

[19] www.wikipedia.org. 